\title{
ASYMPTOTIC BEHAVIOR OF THE SOLUTIONS OF THE SECOND ORDER DIFFERENCE EQUATION
}

\author{
ANDRZEJ DROZDOWICZ AND JERZY POPENDA
}

\begin{abstract}
The second order difference equation
\end{abstract}

$$
\Delta^{2} x_{n}+p_{n} f\left(x_{n}\right)=0
$$

is considered. The results give a necessary and sufficient condition for some solution of (E) to have asymptotic behavior $x_{n} \sim C=$ const. as $n$ approaches infinity.

Introduction. The asymptotic behavior of the solutions of second order differential equations have been considered by R. A. Moore and Z. Nehari [4], W. F. Trench [9], and P. Waltman [10]. The next results for $n$th order nonhomogeneous differential equations was given by T. G. Hallam $[\mathbf{1}, 2]$. Similar problems with regard to second order difference equations were investigated by J. W. Hooker and W. T. Patula [3] and J. Popenda [7].

In this paper the asymptotic behavior of solutions of the second order difference equation

$$
\Delta^{2} x_{n}+p_{n} f\left(x_{n}\right)=0
$$

will be considered. A necessary and sufficient condition for some solution $x$ of $(\mathrm{E})$ to have the asymptotic behavior

$$
\lim _{n \rightarrow \infty} x_{n}=C,
$$

where $C$ is a constant such that $f(C) \neq 0$, will be proved.

Let $N$ denote the set of positive integers and $R$ the set of real numbers. Throughout this paper it will be assumed that $f: R \rightarrow R$ is continuous and $p: N \rightarrow R_{+} \cup\{0\}$.

For a function $a: N \rightarrow R$ we introduce the difference operator $\Delta$ by

$$
\Delta a_{n}=a_{n+1}-a_{n}, \quad \Delta^{2} a_{n}=\Delta\left(\Delta a_{n}\right),
$$

where $a_{n}=a(n), n \in N$. Moreover let $\sum_{j=k}^{k-1} a_{j}=0$. One can observe that if $f$ is definite and finite on $R$ then (E) possesses solutions for any two initial values $x_{1}, x_{2} \in R$.

\section{A necessary condition.}

THEOREM 1. A necessary condition for the existence of a solution $x$ of (E) which possesses asymptotic behavior $(\mathrm{AB})$ is

$$
\sum_{j=1}^{\infty} j p_{j}<\infty \text {. }
$$

Received by the editors November 25, 1985.

1980 Mathematics Subject Classification (1985 Revision). Primary 39A10.

Key words and phrases. Difference equation, asymptotic behavior. 
PROOF. Let $x$ denote a solution of (E) having the property (AB), i.e. $x_{n} \rightarrow C$ for $n \rightarrow \infty$. Then

$$
\Delta x_{n} \rightarrow 0 \text { as } n \rightarrow \infty .
$$

Assume that $f(C)>0$. (The case $f(C)<0$ with some modifications can be considered in a similar way.) The continuity of $f$ implies that there exists $\varepsilon>0$ such that $f(t)>0, t \in I:=[C-\varepsilon, C+\varepsilon]$ for some $\varepsilon>0$. Since $x_{n} \rightarrow C$ as $n \rightarrow \infty$, there exists $n_{1}=N(\varepsilon)$ such that for each $n \geq n_{1}, x_{n} \in[C-\varepsilon, C+\varepsilon]$. Therefore

$$
f\left(x_{n}\right) \geq C_{0}:=\min _{t \in I} f(t)>0 \text { for } n \geq n_{1} \text {. }
$$

Hence

$$
\Delta x_{n}-\Delta x_{k}=-\sum_{j=k}^{n-1} p_{j} f\left(x_{j}\right) \leq-C_{0} \sum_{j=k}^{n-1} p_{j} \quad \text { for } k \geq n_{1} .
$$

Using (1.1) we get

$$
C_{0} \sum_{j=k}^{\infty} p_{j} \leq \Delta x_{k} \quad \text { for } k \geq n_{1} .
$$

Therefore the series $\sum_{j=k}^{\infty} p_{j}$ is convergent. Summing (1.2) over $n$ and tending to infinity with an upper limit we yield

$$
C_{0} \sum_{j=n_{1}}^{\infty} \sum_{i=j}^{\infty} p_{i} \leq C-x_{n_{1}}
$$

From this fact it follows that the series $\sum_{j=n_{1}}^{\infty} \sum_{i=j}^{\infty} p_{i}$ converges. Since

$$
\sum_{j=n_{1}}^{\infty} \sum_{i=j}^{\infty} p_{i}=\sum_{j=n_{1}}^{\infty}\left(j+1-n_{1}\right) p_{j}
$$

the series $\sum_{j=n_{1}}^{\infty}\left(j+1-n_{1}\right) p_{j}$ is also convergent. By observing that

$$
\sum_{j=n_{1}}^{\infty} j p_{j}=\sum_{j=n_{1}}^{\infty}\left(j+1-n_{1}\right) p_{j}+\left(n_{1}-1\right) \sum_{j=n_{1}}^{\infty} p_{j}
$$

we see that the series $\sum_{j=n_{1}}^{\infty} j p_{j}$ is convergent. Q.E.D.

REMARK 1. From (1.2) it follows that $\Delta x_{k} \geq 0$ for $k \geq n_{1}$. Therefore the solution $x_{n}$ is increasing for $n \geq n_{1}$. We see that $x_{l} \leq C$ for $l \geq n_{1}$. This result means that if $f(C)>0$ then the solution of (E) which possesses the asymptotic behavior (AB) monotonically approaches $C$ from below. If $f(C)<0$, then $x_{n}$ must monotonically tend to $C$ from above.

\section{A sufficient condition.}

THEOREM 2. For every $k \in N$ let

$(*) i_{R}+p_{k} f: R \rightarrow R$ be a surjection ( $i_{R}$ denotes an identity function on $R$ ).

$A$ sufficient condition for the existence of a solution $x$ of $(\mathrm{E})$ which possesses the asymptotic behavior $(\mathrm{AB})$ is $(\mathrm{NS})$.

Proof. The cases $C>0$ and $f(C)>0$ will be considered. (The other cases, i.e. $C<0$ or $f(C)<0$, with some modifications can be shown in a similar way.) 
Let (NS) hold. Hence

$$
\lim _{n \rightarrow \infty} \sum_{j=n}^{\infty} j p_{j}=0
$$

One can observe that the sequence $\left\{\sum_{j=n}^{\infty} j p_{j}\right\}_{n=1}^{\infty}$ is nonincreasing. Analogous to the proof of Theorem 1 there exists an interval $I=[C-\varepsilon, C+\varepsilon]$ such that $f(t)>0$, $t \in I$ for some $\varepsilon>0$. Denoting $C_{1}:=\max _{t \in I^{-}} f(t)$, where $I^{-}=[C-\varepsilon, C]$ from (2.1), we obtain

$$
C_{1} \sum_{j=n}^{\infty} j p_{j} \leq \varepsilon \quad \text { for all } n \geq N(\varepsilon)
$$

Let us set

$$
n_{2}=\min \left\{n \in N: C_{1} \sum_{j=n}^{\infty} j p_{j} \leq \varepsilon\right\} .
$$

Let $l_{\infty}$ denote the Banach space of bounded sequences $x=\left\{h_{i}\right\}_{i=1}^{\infty}$ with norm $\|x\|=\sup _{i \geq 1}\left|h_{i}\right|$. Moreover let us define the set $T \subset l_{\infty}$ in the following way:

$$
x=\left\{h_{i}\right\}_{i=1}^{\infty} \in T \quad \text { if }\left\{\begin{array}{l}
h_{k}=C \text { for } k=1,2, \ldots, n_{2}-1, \\
h_{k} \in I_{k}^{-} \quad \text { for } k \geq n_{2}
\end{array}\right.
$$

where

$$
I_{k}^{-}:=\left[C-C_{1} \sum_{j=k}^{\infty} j p_{j}, C\right], \quad k \geq n_{2} .
$$

It is easy to show that $T$ is bounded, convex and closed in $l_{\infty}$. We will show that $T$ is compact. Set $\operatorname{diam}[a, b]=b-a ; a, b \in R$. By (NS) it follows that $\operatorname{diam} I_{n}^{-} \rightarrow 0$ for $n \rightarrow \infty$. Choose any $\varepsilon_{1}>0$. If $\varepsilon_{1}$ is such that diam $I_{n_{2}}^{-}<\varepsilon_{1}$, then the element $v=\{C, C, C, \ldots\} \in l_{\infty}$ is an $\varepsilon_{1}$-net. The case diam $I_{n_{2}}^{-} \geq \varepsilon_{1}$ will be considered. Let $n_{3} \geq n_{2}$ be such that $\operatorname{diam} I_{n_{3}}^{-} \geq \varepsilon_{1}$ and $\operatorname{diam} I_{n_{3}+1}^{-}<\varepsilon_{1}$. (Everyone can find $n_{3}$ because diam $I_{n}^{-} \rightarrow 0$ for $n \rightarrow \infty$.) Then it is easy to show that the set of elements of the space $l_{\infty}$ in the form

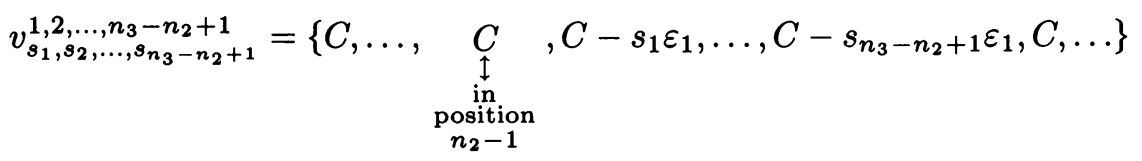

where

$$
s_{i}=0,1, \ldots, r_{i}:=\operatorname{En}\left[\frac{\operatorname{diam} I_{n_{2}+i-1}^{-}}{\varepsilon_{1}}\right]+1, \quad i=1,2, \ldots, n_{3}-n_{2}+1,
$$

to set up an $\varepsilon_{1}$-net. (En denotes an entire function.) One can observe that

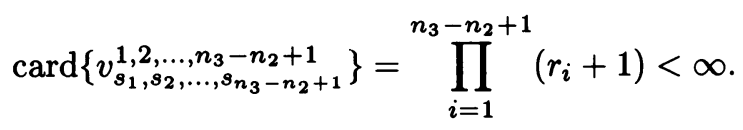

Hence the $\varepsilon_{1}$-net is finite and by the Hausdorff theorem $T$ is compact.

Let us define the operator $A$ on $T$ in the following way:

$$
A x=y=\left\{b_{1}, b_{2}, \ldots, b_{n_{2}-1}, b_{n_{2}}, \ldots, b_{k}, \ldots\right\},
$$


where

$$
b_{n}=\left\{\begin{array}{l}
C \quad \text { for } n=1,2, \ldots, n_{2}=1 \\
C-\sum_{j=n}^{\infty}(j+1-n) p_{j} f\left(h_{j}\right) \quad \text { for } n \geq n_{2}
\end{array}\right.
$$

We will show that $A$ is a function from $T$ to $T$. By observing that $I_{k}^{-} \subset I^{-}$it follows that $0<f\left(h_{k}\right) \leq C_{1}$ for $k \geq n_{2}$. For $j \geq k$ one obtains the inequality

$$
0<(j+1-k) p_{j} f\left(h_{j}\right) \leq j p_{j} f\left(h_{j}\right) \leq C_{1} j p_{j} .
$$

Hence

$$
C \geq C-\sum_{j=k}^{\infty}(j+1-k) p_{j} f\left(h_{j}\right) \geq C-C_{1} \sum_{j=k}^{\infty} j p_{j} .
$$

Thus $b_{k} \in I_{k}^{-}$for $k \geq n_{2}$. Therefore $y \in T$.

Next we will show that $A$ is continuous. Since $f$ is continuous on $R$, it is uniformly continuous on $I^{-}$. Hence for each $\varepsilon_{2}>0$ there exists $\delta_{1}>0$ such that the condition $\left|t_{1}-t_{2}\right|<\delta_{1}$ implies $\left|f\left(t_{1}\right)-f\left(t_{2}\right)\right|<\varepsilon_{2}$. Consider the sequence $\left\{x^{m}\right\}_{m=1}^{\infty}, x^{m} \in T$, such that

$$
\left\|x^{m}-x^{0}\right\| \rightarrow 0 ; \quad \text { i.e., } \sup _{n \geq 1}\left|h_{n}^{m}-h_{n}^{0}\right| \rightarrow 0, \quad \text { as } m \rightarrow \infty .
$$

From (2.2) it follows that there exists $n_{3}=N\left(\delta_{1}\right)$ such that

$$
\left\|x^{m}-x^{0}\right\|<\delta_{1} ; \quad \text { i.e., } \sup _{n \geq 1}\left|h_{n}^{m}-h_{n}^{0}\right|<\delta_{1} \quad \text { for } m \geq n_{3} .
$$

Hence

$$
\underset{m \geq n_{3}}{\forall} \underset{i \in N}{\forall}\left|h_{i}^{m}-h_{i}^{0}\right|<\delta_{1}
$$

Then for $m \geq n_{3}$

$$
\begin{aligned}
\left\|A x^{m}-A x^{0}\right\| & =\sup _{n \geq 1}\left|b_{n}^{m}-b_{n}^{0}\right| \\
& =\sup _{n \geq n_{2}}\left|\sum_{j=n}^{\infty}(j+1-n) p_{j} f\left(h_{j}^{m}\right)-\sum_{j=n}^{\infty}(j+1-n) p_{j} f\left(h_{j}^{0}\right)\right|,
\end{aligned}
$$

where $b^{0}=A x^{0}$ and $b^{m}=A x^{m}$.

Since the series $\sum_{j=n}^{\infty}(j+1-n) p_{j} f\left(h_{j}^{m}\right), \sum_{j=n}^{\infty}(j+1-n) p_{j} f\left(h_{j}^{0}\right)$ are convergent,

$$
\left\|A x^{m}-A x^{0}\right\| \leq \varepsilon_{2} \sum_{j=n_{2}}^{\infty}\left(j+1-n_{2}\right) p_{j}, \quad m \geq n_{3} .
$$

Hence $A$ is continuous.

By the Schauder fixed point theorem [8] there exists a solution in $T$ of the equation $x=A x$. Let $z=\left\{d_{1}, d_{2}, \ldots, d_{n_{2}-1}, d_{n_{2}}, \ldots\right\}$ denote such a solution. Since $z \in T$, it can be written as follows:

$$
z=\left\{C, C, \ldots, C, d_{n_{2}}, d_{n_{2}+1}, \ldots\right\}
$$


and

$$
\begin{aligned}
A z=\left\{C, C, \ldots, C, C-\sum_{j=n_{2}}^{\infty}\left(j+1-n_{2}\right) p_{j} f\left(d_{j}\right),\right. & \\
& \left.C-\sum_{j=n_{2}+1}^{\infty}\left(j-n_{2}\right) p_{j} f\left(d_{j}\right), \ldots\right\} .
\end{aligned}
$$

Therefore

$$
d_{n}=C-\sum_{j=n}^{\infty}(j+1-n) p_{j} f\left(d_{j}\right) \text { for } n \geq n_{2}
$$

Applying the operator $\Delta$ to $(2.3)$ we yield

$$
\Delta d_{n}=\sum_{j=n}^{\infty} p_{j} f\left(d_{j}\right) \text { for } n \geq n_{2} .
$$

Hence $\Delta^{2} d_{n}=-p_{n} f\left(d_{n}\right)$ holds for $n \geq n_{2}$. This means that the sequence $\left\{d_{n}\right\}_{n=1}^{\infty}$ fulfills the equation (E) but for $n \geq n_{2}$ only.

We now prove the existence of the solution $\left\{x_{n}\right\}_{n=1}^{\infty}$ of $(\mathrm{E})$ such that $x_{n}=d_{n}$ for $n \geq n_{2}$.

One can observe that $(\mathrm{E})$ can be rewritten as

$$
x_{n}+p_{n} f\left(x_{n}\right)=-x_{n+2}+2 x_{n+1} \text {. }
$$

If $n=n_{2}-1$ we get

$$
x_{n_{2}-1}+p_{n_{2}-1} f\left(x_{n_{2}-1}\right)=-x_{n_{2}+1}+2 x_{n_{2}} .
$$

But we demand for $x_{n}$ to be equal to $d_{n}$ for $n \geq n_{2}$.

From (2.4) we obtain

$$
x_{n_{2}-1}+p_{n_{2}-1} f\left(x_{n_{2}-1}\right)=-d_{n_{2}+1}+2 d_{n_{2}} .
$$

By (*) it follows that the equation

$$
x+p_{n_{2}-1} f(x)=-d_{n_{2}+1}+2 d_{n_{2}}
$$

possesses solutions. Let us denote one of them by $x_{n_{2}-1}$. Analogously we can calculate $x_{n_{2}-2}, x_{n_{2}-3}, \ldots, x_{2}, x_{1}$ one after the other. Consequently we get the sequence which fulfills (2.4), i.e. which also fulfills (E). Moreover this sequence is identical to $\left\{d_{n}\right\}_{n=1}^{\infty}$ for $n \geq n_{2}$ and it has the asymptotic behavior (AB) because $\lim _{n \rightarrow \infty} d_{n}=C$. Q.E.D.

REMARK 2. One can observe that if $f$ is bounded on $R$ or fulfills the condition $x f(x)>0$ for $x \neq 0$ then condition (*) is satisfied. From the proof of Theorem 2 we can deduce that $(*)$ may be weakened as follows:

$$
i_{R}+p_{k} f: R \rightarrow R \text { for } k<n_{2}, k \in N .
$$

REMARK 3. If the assumptions of Theorem 2 hold then analogously an existence of a solution of the equation

$$
\Delta^{2} x_{n}+p_{n+k} f\left(x_{n+k}\right)=0, \quad k \geq 1,
$$


having the asymptotic behavior (AB) may be proved. In this case the operator $A$ similar to the above but with

$$
b_{n}=C-\sum_{j=n+k}^{\infty}(j+1-n-k) p_{j} f\left(h_{j}\right) \quad \text { for } x=\left\{h_{i}\right\}_{i=1}^{\infty} \in T
$$

should be defined.

REMARK 4. If (E) possesses a solution $x$ such that $\lim _{n \rightarrow \infty} x_{n}=C$ then equation (E) has a solution with $\lim _{n \rightarrow \infty} x_{n}=C_{2}$, where $C_{2} \in(C-\varepsilon, C+\varepsilon) \subset I$.

REMARK 5. If for some $C, f(C)=0$, then independently of the form of $p$, equation (E) has a solution with (AB). It has the form $x_{n}=C$ for each $n \geq 1$. Conversely, if, for each $n \geq n_{2}, x_{n}=C$ is the solution of (E) then $p_{n} f(C)=0$ for $n \geq n_{2}$. Hence $f(C)=0$ or $p_{n}=0$ for each $n \geq n_{2}$. For the second case $\left(p_{n}=0\right)$ the condition $\sum_{j=1}^{\infty} j p_{j}<\infty$ obviously holds.

EXAMPLE. The special case $f(x)=x$ and $k=1$ will be studied. In this case the equation $\left(\mathrm{E}_{\mathrm{k}}\right)$ can be written in the following two equivalent forms:

$$
\Delta^{2} x_{n}+p_{n+1} x_{n+1}=0, \quad x_{n+2}-q_{n} x_{n+1}+x_{n}=0,
$$

where $q_{n}=2-p_{n+1}, n \in N$. If $q_{n}<2, n \in N$ and $\sum_{j=2}^{\infty}\left(2-q_{j-1}\right) j<\infty$ then $\left(\mathrm{E}_{1}\right)$ possesses a solution which asymptotically approaches any positive constant.

Analogously in the case $k=2$ one obtains the equation

$$
x_{n+2}-2 q_{n} x_{n+1}+q_{n} x_{n}=0,
$$

where $q_{n}=1 /\left(p_{n+2}+1\right)$.

If $0<q_{n}<1$ and $\sum_{j=3}^{\infty}\left(1 / q_{j-1}-1\right) j<\infty$ then $\left(\mathrm{E}_{2}\right)$ possesses a solution which asymptotically approaches any positive constant.

\section{REFERENCES}

1. T. G. Hallam, Asymptotic behavior of the solutions of an nth order nonhomogeneous ordinary differential equation, Trans. Amer. Math. Soc. 122 (1966), 177-196.

2. __ Asymptotic behavior of the solutions of a nonhomogeneous singular equation, $\mathrm{J}$. Differential Equations 3 (1967), 135-152.

3. J. W. Hooker and W. T. Patula, A second order nonlinear difference equation; oscillation and asymptotic behavior, J. Math. Anal. Appl. 91 (1983), 9-29.

4. R. A. Moore and Z. Nehari, Nonoscillation theorems for a class of nonlinear differential equations, Trans. Amer. Math. Soc. 93 (1959), 30-52.

5. W. T. Patula, Growth and oscillation properties of second order linear difference equations, SIAM J. Math. Anal. 10 (1979), 55-61.

6. __ Growth, oscillation and comparison theorems for second order linear difference equations, SIAM J. Math. Anal. 10 (1979), 1272-1279.

7. J. Popenda and J. Werbowski, On the asymptotic behaviour of the solutions of difference equations of second order, Comment. Math. 22 (1980), 135-142.

8. J. Schauder, Zur Theorie stetiger Abbildungen in Funktionalraumen, Math. Z. 26 (1927), 47-65.

9. W. F. Trench, On the asymptotic behavior of solutions of second order linear differential equations, Proc. Amer. Math. Soc. 14 (1963), 12-14.

10. P. Waltman, On the asymptotic behavior of solutions of a nonlinear equation, Proc. Amer. Math. Soc. 15 (1964), 918-923.

Institute of Mathematics, Technical University of Poznań, ul. Piotrowo 3A, 60-965 POZNAŃ, POLAND 